\title{
An Adaptively Accelerated Bayesian Deblurring Method with Entropy Prior
}

\author{
Manoj Kumar Singh, ${ }^{1}$ Uma Shanker Tiwary, ${ }^{2}$ and Yong-Hoon Kim ${ }^{1}$ \\ ${ }^{1}$ Sensor System Laboratory, Department of Mechatronics, Gwangju Institute of Science and Technology (GIST), \\ 1 Oryong-dong, Buk-gu, Gwangju-500 712, South Korea \\ ${ }^{2}$ Indian Institute of Information Technology, Allahabad 211-012, India
}

Correspondence should be addressed to Yong-Hoon Kim, yhkim@gist.ac.kr

Received 28 August 2007; Revised 15 February 2008; Accepted 4 April 2008

Recommended by C. Charrier

\begin{abstract}
The development of an efficient adaptively accelerated iterative deblurring algorithm based on Bayesian statistical concept has been reported. Entropy of an image has been used as a "prior" distribution and instead of additive form, used in conventional acceleration methods an exponent form of relaxation constant has been used for acceleration. Thus the proposed method is called hereafter as adaptively accelerated maximum a posteriori with entropy prior (AAMAPE). Based on empirical observations in different experiments, the exponent is computed adaptively using first-order derivatives of the deblurred image from previous two iterations. This exponent improves speed of the AAMAPE method in early stages and ensures stability at later stages of iteration. In AAMAPE method, we also consider the constraint of the nonnegativity and flux conservation. The paper discusses the fundamental idea of the Bayesian image deblurring with the use of entropy as prior, and the analytical analysis of superresolution and the noise amplification characteristics of the proposed method. The experimental results show that the proposed AAMAPE method gives lower RMSE and higher SNR in 44\% lesser iterations as compared to nonaccelerated maximum a posteriori with entropy prior (MAPE) method. Moreover, AAMAPE followed by wavelet wiener filtering gives better result than the state-of-the-art methods.
\end{abstract}

Copyright (c) 2008 Manoj Kumar Singh et al. This is an open access article distributed under the Creative Commons Attribution License, which permits unrestricted use, distribution, and reproduction in any medium, provided the original work is properly cited.

\section{INTRODUCTION}

Image deblurring, process of restoration of an image from its blurred and noisy version, is an enduring linear inverse problem and is encountered in many application areas such as in remote sensing, medical imaging, seismology, and astronomy [1-3]. Generally, many linear inverse problems are ill-conditioned since the inverse of linear operators either does not exist or is nearly singular yielding highly noise sensitive solutions. The methods for solving ill-conditioned linear inverse problems can be classified into the following two general categories: (a) methods based on regularization [2-4] and (b) methods based on Bayesian theory $[2,4,5]$.

The main idea of regularization and Bayesian approach is the use of a priori information expressed by prior term. The prior term gives a higher score to most likely images, hence, helps in selection of single image from many images, which fits the noisy and blurred observation. However, modeling a prior for real-word images is not a trivial and subjective matter. Many directions for prior modeling have been proposed such as derivative energy in the Wiener filter [3], the compound Gauss-Markov random field $[6,7]$, the Markov random fields (MRFs) with nonquadratic potentials $[2,8,9]$, entropy $[1,3,4,10-12]$, and heavy-tailed densities of images in wavelet domain [13]. An excellent review on image deblurring methods is available in [14].

The weak points in the regularization setup and the compound Gauss-Markov random field in the Bayesian setup were conceived to model piecewise-smooth images. By detecting boundaries between two smooth regions with discrete random variables, the so-called line-field, these priors improve the modeling accuracy near the edge in comparison to the classical quadratic one. The MRFs have been widely used to model local smoothness in images [15], but it leads to computationally intensive deblurring methods. In absence of any prior information, smoothness or texture, about the original image entropy is considered as the best choice to define prior term. 
In this paper, we describe the maximum a posteriori with entropy prior (MAPE) method for image deblurring in Bayesian framework. This method is nonlinear and solved iteratively. However, it has the drawbacks of slow convergence and being intensive in computation. Many techniques for accelerating the iterative methods for faster convergence have been proposed $[1,16-21]$. These acceleration methods can also be used for ensuring the acceleration of the MAPE method. All these acceleration techniques use correction term which is computed in each iteration and added to the result obtained in the previous iteration. In most of these acceleration methods, the correction term is obtained by multiplying gradient of objective function with acceleration parameter. Acceleration methods given in [17-19] use the line search approach to find acceleration parameter to maximize the objective function (likelihood/log-likelihood function) at each iteration. It speeds up the iterative method by a factor of $2 \sim 5$, but requires a prior limit on acceleration parameter to prevent the divergence. Maximizing a function in the direction of the gradient is termed as steepest ascent, and minimizing a function (in the negative gradient direction) is called steepest descent. The main problem with gradient-based methods is the selection of optimal acceleration step. Large acceleration step speeds up the algorithms, but it may introduce error. If error is amplified during iteration, it can lead to instability. Thus, gradientbased methods require an acceleration step followed by a correction step to ensure the stability. This correction step reduces the gain obtained by the acceleration step.

A gradient search method proposed in [20], known as conjugate gradient (CG), method is better than the above discussed methods. This approach has also been proposed by the authors of [22] as well as $[17,21]$. The CG method requires gradient of the objective function and an efficient line search technique. But drawback of CG method is that several function evaluations are needed in order to accurately maximize/minimize objective function, and in many cases the objective function and its gradient are not trivial.

One of our objectives in this paper is to give simple and efficient method which overcomes difficulties in previously proposed methods. In order to cope with the problems of earlier accelerated methods, the proposed AAMAPE method requires minimum information about the iterative process. Our method uses the multiplicative correction term instead of using additive correction term. Multiplicative correction term is obtained from derivative of conditional log-likelihood. We use an exponent on multiplicative correction as an acceleration parameter which is computed adaptively in each iteration, using first-order derivatives of deblurred image from previous two iterations. The positivity of pixel intensity in the proposed acceleration method is automatically ensured since multiplicative correction term is always positive, while in other acceleration methods based on additive correction term, the positivity is enforced manually at the end of iteration.

Another important objective of this paper is to analyze the superresolution and the nature of noise amplification in the proposed AAMAPE method. Superresolution means restoring the frequency beyond the diffraction limit. It is often bandied about nonlinear methods that they have superresolution capability, but very limited insight for superresolution is available. In [23], an analysis of superresolution is performed assuming that the point spread function (PSF) of the system and the object intensity are Gaussian function. In this paper, we present general analytical interpretation of superresolving capability of the proposed AAMAPE method and confirmed it experimentally.

It is a well-known fact about the nonlinear methods based on maximum likelihood that the restored images begin to deteriorate after certain number of iterations. This deterioration is due to the noise amplification in successive iterations. Due to the nonlinearity, an analytical analysis of the noise amplification for a nonlinear method is difficult. In this paper, we investigate the process of noise amplification qualitatively for the proposed AAMAPE method.

The rest of the paper is organized as follows. Section 2 describes the observation model and the proposed AAMAPE method. Section 3 presents analytical analysis for the superresolution and noise amplification for the proposed method. Experimental results and discussion are given in Section 4. The conclusion is presented in Section 5 which is followed by references.

\section{BAYESIAN FRAMEWORK FOR IMAGE DEBLURRING}

\subsection{Observation model}

Consider an original image, $x$ of size $M \times N$, blurred by shiftinvariant point spreading function (PSF), $h$, and corrupted by Poisson noise. Observation model for the blurring in case of Poisson noise is given as

$$
y=P((h \otimes x)(z))
$$

where $P$ denotes the Poisson distribution, $\otimes$ is convolution operator, $z$ is defined on a regular $M \times N$ lattice $Z=$ $\left\{m_{1}, m_{2}: m_{1}=1,2, \ldots, M, m_{2}=1,2, \ldots, N\right\}$. Alternatively, observation model (1) can be expressed as

$$
y(z)=(h \otimes x)(z)+n(z)
$$

where $n$ is zero-mean with variance $\sigma_{n}^{2}(z)=\operatorname{var}\{n(z)\}=$ $(h \otimes x)(z)$. Blurred and noisy image, $y$, has mean, $E\{y(z)\}=$ $(h \otimes x)(z)$, and variance, $\sigma_{y}^{2}(z)=(h \otimes x)(z)$. Thus, the observation variance $\sigma_{y}^{2}(z)$ is signal dependent and, consequently, spatially variant. For mathematical simplicity, observation model (2) can be expressed in matrix-vector form as follows:

$$
\bar{y}=\bar{H} \bar{x}+\bar{n}
$$

where $\bar{H}$ is the blurring operator of size $M N \times M N$ corresponding PSF $h ; \bar{x}, \bar{y}$, and $\bar{n}$ are vectors of size $M N \times 1$ containing the original image, observed image, and sample of noise, respectively, arranged in a column lexicographic ordering. The aim of image deblurring is to recover an original image, $\bar{x}$, from its degraded version $\bar{y}$. 


\subsection{Entropy as a prior distribution}

The basic idea of Bayesian framework is to incorporate the prior information, about the desired image. The prior information is included using a priori distribution. The a priori distribution $p(\bar{x})$ is defined using entropy as [12]

$$
p(\bar{x})=\exp (\lambda E(\bar{x})), \quad \lambda>0
$$

where $E(\bar{x})$ is the entropy of the original image $\bar{x}$. The role of entropy in defining the a prioridistribution has been in discussion for four decades [24-26], and researcher proposed many entropy functions [27]. Frieden first used the Shannon form of entropy in the context of image reconstruction [25]. We also use the Shannon entropy, and, it is given as follows:

$$
E(\bar{x})=-\sum_{i} x_{i} \log x_{i}
$$

It is important to note that in spite of the large volume of literature justifying the choice of entropy as a priori distribution, the entropy of an image does not have the same firm conceptual foundation as in statistical mechanics [14]. Trussell [28] has found a relationship between the use of entropy as a prior in a maximum aposteriori (MAP) image restoration and a simple maximum entropy image restoration with constraints for the Gaussian noise case [3]. In the case of Gaussian noise first-order approximation of MAP with Shannon entropy gives the well-known Tikhonov method of image restoration [3].

\subsection{Accelerated MAP with entropy prior}

When $\bar{n}$ is zero in (3), we consider only blurring, the expected value at the $i$ th pixel in the blurred image is $\sum_{j} h_{i j} x_{j}$. Where $h_{i j}$ is $(i, j)$ th element of $\bar{H}$ and $x_{j}$ is the $j$ th element of $\bar{x}$. Because of Poison noise, the actual ith pixel value, $y_{i}$, in $\bar{y}$ is one realization of Poisson distribution with mean $\sum_{j} h_{i j} x_{j}$. Thus, we have the following relation:

$$
p\left(y_{i} / \bar{x}\right)=\frac{\left(\sum_{j} h_{i j} x_{j}\right)^{y_{i}} e^{\left(-\sum_{j} h_{i j} x_{j}\right)}}{y_{i} !} .
$$

Each pixel in blurred and noisy image $\bar{y}$ is realized by an independent Poisson process. Thus, the likelihood of getting noisy and blurred image $\bar{y}$ is given by

$$
p(\bar{y} / \bar{x})=\prod_{i}\left(\frac{\left(\sum_{j} h_{i j} x_{j}\right)^{y_{i}} e^{\left(-\sum_{j} h_{i j} x_{j}\right)}}{y_{i} !}\right) .
$$

From Bayes's theorem, we get a posteriori probability $p(\bar{x} / \bar{y})$ of $\bar{x}$ for given $\bar{y}$ as

$$
p(\bar{x} / \bar{y})=\frac{p(\bar{y} / \bar{x})}{p(\bar{y})}
$$

MAPE method with flux conservation for image deblurring seeks an approximate solution of (1) that maximizes the a posteriori probability $p(\bar{x} / \bar{y})$ or $\log p(\bar{x} / \bar{y})$, subject to the constraint of flux conservation, $\sum_{j} x_{j}=\sum_{j} y_{j}=N$. We consider the maximization of the following function:

$$
L(\bar{x}, \mu)=\log p(\bar{x} / \bar{y})-\mu\left(\sum_{j} x_{j}-N\right)
$$

where $\mu$ is the Lagrange multiplier for flux conservation. On substitution of $p(\bar{x} / \bar{y})$ from (8) into (9), we get

$$
L(\bar{x}, \mu)=\log p(\bar{y} / \bar{x})+\log p(\bar{x})-\log p(\bar{y})-\mu\left(\sum_{j} x_{j}-N\right) .
$$

Substituting the $p(\bar{x}), p(\bar{y} / \bar{x})$ from $(5),(7)$ in (10), we get the following:

$$
\begin{aligned}
L(\bar{x}, \mu)= & \sum_{i}\left\{-\sum_{j} h_{i j} x_{j}+y_{i} \log \left(\sum_{l} h_{i l} x_{l}\right)\right\} \\
& -\lambda \sum_{j} x_{j} \log x_{j}-\log p(\bar{y})-\mu\left(\sum_{j} x_{j}-N\right) .
\end{aligned}
$$

From $\partial L / \partial x_{j}=0$ we get the following relation:

$$
1+\rho \mu=\rho \sum_{i} h_{i j}\left(\frac{y_{i}}{\sum_{l} h_{i l} x_{l}}\right)-\rho-\log \left(x_{j}\right)
$$

where $\rho=\lambda^{-1}$. Adding a positive constant $C$ and introducing an exponent $q$ on both sides of (12), we get the following relation:

$$
(1+\rho \mu+C)^{q}=\left\{\rho \sum_{i} h_{i j}\left(\frac{y_{i}}{\sum_{l} h_{i l} x_{l}}\right)-\rho-\log \left(x_{j}\right)+C\right\}^{q} .
$$

Equation (13) is nonlinear, and is solved iteratively. Multiply both sides of (13) by $x_{j}$, we arrive on the following iterative procedure:

$$
x_{j}^{k+1}=A x_{j}^{k}\left\{\rho \sum_{i} h_{i j}\left(\frac{y_{i}}{\sum_{l} h_{i l} x_{l}^{k}}\right)-\rho-\log \left(x_{j}^{k}\right)+C\right\}^{q},
$$

where $A=[\overline{1}+\rho \mu+C]^{-q}$. For ensuring the nonnegativity of $x_{j}^{k}$ and the computation of $\log \left(x_{j}^{k}\right)$ in the next iteration a constant $c>\rho+\log \left(x_{j}^{k}\right)$ added both sides of (14). By generalizing (14), we get the following:

$$
\bar{x}^{k+1}=A \bar{x}^{k}\left[\rho \bar{H}^{T}\left(\bar{y} / \bar{H} \bar{x}^{k}\right)-\rho-\log \left(\bar{x}^{k}\right)+C\right]^{q},
$$

where superscript $T$ denotes transpose of matrix, and $\left(\bar{y} / \bar{H} \bar{x}^{k}\right)$ denotes the vector obtained by component-wise division of $\bar{y}$ by $\bar{H} \bar{x}^{k}$. Likewise, component-wise multiplication of the vector $\bar{x}^{k}$ and vector obtained from square bracket of (15). In order to show that the iterative procedure (15) has fixed point, we proved that it satisfies the condition of contraction mapping (Section 2.6). 
In further discussions, we use the word "correction factor" to refer to the expression in square bracket of (15). The constant $A$ is known if $\rho, \mu$ are known and their values must be such that at convergence $\sum_{j} x_{j}=N$ holds. Accordingly, in the iteration (14), the constant $A$ is recalculated at each iteration so that $\sum_{j} x_{j}^{k}=N$ satisfied. Summing both sides of (14) over all pixel values, we get

$$
\sum_{j} x_{j}^{k+1}=A \sum_{j} x_{j}^{k}\left\{\rho \sum_{i} h_{i j}\left(\frac{y_{i}}{\sum_{l} h_{i l} x_{l}^{k}}\right)-\rho-\log \left(x_{j}^{k}\right)+C\right\}^{q} .
$$

Using flux conservation constraint, $\sum_{j} x_{j}^{k+1}=N$, in (16) we get the following expression for $A$ :

$$
\begin{aligned}
A & =A(k) \\
& =N\left[\sum_{j} x_{j}^{k}\left\{\rho \sum_{i} h_{i j}\left(\frac{y_{i}}{\sum_{l} h_{i l} x_{l}^{k}}\right)-\rho-\log x_{j}^{k}+C\right\}^{q}\right]^{-1} .
\end{aligned}
$$

From (17), we see that the constant $A$ does not depend on the Lagrange multiplier $\mu$. Thus, iterative procedure (14), (15) does not depend on $\mu$.

We observed that iteration given in (15) converges only for some values of $q$ lying between 1 and 3. Large values of $q(\approx 3)$ may give faster convergence but with the increased risk of instability. Small values of $q(\approx 1)$ lead to slow convergence with reduced risk of instability. Between these two extremes, the adaptive selection of exponent $q$ provides means for achieving faster convergence while ensuring stability. Thus, (15) with an adaptive selection of exponent $q$ leads to the AAMAPE method. An empirical method for adaptive selection of $q$ is discussed in Section 2.5. Putting $q=1$ in (15), we get the following equation:

$$
\bar{x}^{k+1}=A \bar{x}^{k}\left[\rho \bar{H}^{T}\left(\bar{y} / \bar{H} \bar{x}^{k}\right)-\rho-\log \left(\bar{x}^{k}\right)+C\right] .
$$

Equation (18) is nonaccelerated maximum a posteriori with entropy (MAPE) method for image deblurring. This can be derived from (12).

As $\lambda \rightarrow 0$ in (11), maximization of $L(\bar{x}, \mu)$ amounts to maximize the first term of (11), equivalently $\log (\bar{y} / \bar{x})$, with respect to $\bar{x}$. This leads to well-known Lucy-Richardson method, where flux conservation and nonnegativity is automatic. Hence, for large $\rho$, this method behaves like the Lucy-Richardson method and can be expected to be unstable in presence of noise. For large value of $\lambda$ the prior probability term becomes dominant in $L(\bar{x}, \mu)$ and, hence, prior probability is maximized when $\bar{x}$ is a uniform image. Thus, small value of $\rho$ imposes high degree of uniformity in the restored image, and, hence, smoothing of high frequency component. The optimal value of $\rho$ is obtained by trial and error. Starting with a small value for $\rho$ and iterating to convergence, the procedure must be repeated with larger values of $\rho$ until a value is found which sufficiently sharpens the image at convergence and without undue noise degradation.

\subsection{Nonnegativity in AAMAPE}

It is reported that disallowing the negative pixel intensity strongly suppresses the artifact, increases the resolution, and may speed up the convergence of the algorithm [14]. Nonnegativity is a necessary restriction for almost all kind of images. In many algorithms, nonnegativity constraint is imposed either by change of variable or replacing negative values to some positive value at the end of each iteration.

In accelerated MAPE (15), for $\bar{x}^{k}>0$ the first term, $\rho \bar{H}^{T}\left(\bar{y} / \bar{H} \bar{x}^{k}\right)$, is nonnegative and selection of constant $C>$ $\left(\rho+\log \left(\bar{x}^{k}\right)\right)$ gives the correction factor and constant $A$, which is given by (17), is always positive. Thus, in accelerated MAPE the nonnegativity of intensity, $\bar{x}^{k+1}>0$, is automatically guaranteed.

\subsection{Adaptive selection of $q$}

It is found that the choice of $q$ in (15) mainly depends on the noise, $\bar{n}$, and its amplification during iterations. If noise is high, smaller value of $q$ is selected and vice-versa. The convergence speed of the proposed method depends on the choice of the parameter $q$. Drawback of this accelerated form of MAPE is that the selection of exponent $q$ has to be done manually by trial and error. We overcome this serious limitation by proposing a method by which $q$ is computed adaptively as iterations proceed. We proposed an expression for $q$ based on an empirical observation as follows:

$$
q(k+1)=\exp \left(\frac{\left\|\nabla \bar{x}^{k}\right\|}{\left\|\nabla \bar{x}^{k-1}\right\|}\right)-\frac{\left\|\nabla \bar{x}^{2}\right\|}{\left\|\nabla \bar{x}^{1}\right\|}
$$

where $\nabla \bar{x}^{k}$ stands for first-order derivative of $\bar{x}^{k}$ and $\|\cdot\|$ denotes the $L_{2}$ norm. Main idea in using first-order derivative is to utilize the sharpness of image. Due to blurring the image becomes smooth, sharpness decreases, and edges are lost or become weak. Debluring makes image nonsmooth, and increases the sharpness. Hence, the sharpness of deblurred image increases as iterations proceed. And the ratio $\left\|\nabla x^{k}\right\| /\left\|\nabla x^{k-1}\right\|$ converges to one as the number of iteration increases. For different levels of blur and different classes of images, it has been found by experiments that the ratio $\left\|\nabla x^{2}\right\| /\left\|\nabla x^{1}\right\|$ lying between 1 and 1.5. AAMAPE emphasizes speed at the beginning stages of iterations by forcing $q$ around three. At the start of iteration, when the exponential term in (19) is greater than three, the second term, $\left\|\nabla x^{2}\right\| /\left\|\nabla x^{1}\right\|$, limits the value of $q$ within three to prevent divergence. As iterations proceed the second term forces $q$ toward the value of one which leads to stability of iteration. By using the exponent, $q$, the method emphasizes speed at the beginning stages and the stability at later stages of iteration. Thus, selecting $q$ given by (19) for iterative solution (15) gives AAMAPE. In order to initialize the AAMAPE, first two iterations are computed using some fixed value of $q(1 \leq q \leq 3)$. In order to avoid instability at the starting of iteration, $q=1$ is preferable choice. 


\subsection{Contraction mapping for AAMAPE}

In order to show that the iterative procedure (15) gives the solution of (13), we prove that (15) provides contraction mapping $[29,30]$. A function $f$ on complete metric space $(X, \psi)$ is said to have contracting property, if for any $x^{\prime}, x^{\prime \prime}$ in $X$ the inequality

$$
\psi\left(f\left(x^{\prime}\right), f\left(x^{\prime \prime}\right)\right) \leq c \psi\left(x^{\prime}, x^{\prime \prime}\right)
$$

holds, for some fixed $0<c<1$.

Equation (15) can be considered as a function from $R^{n}$ to $R^{n}$, where $n=M N$ and $R$ takes values in $[0, G]$, and $G$ is the maximum gray code value. For (15), contracting property holds, if for any $\bar{x}^{k_{1}}, \bar{x}^{k_{2}}$ in $R^{n}$ there exists a constant $0<c^{\prime}<1$ such that following inequality holds

$$
d\left(\bar{x}^{k_{2}+1}, \bar{x}^{k_{1}+1}\right) \leq c^{\prime} d\left(\bar{x}^{k_{2}}, \bar{x}^{k_{1}}\right),
$$

where $d$ is Euclidean distance in $R^{n}$.

In order to make mathematical steps simple and tractable, we rewrite (15) using (A.1) as follows:

$$
\bar{x}^{k+1}=A(k) \bar{x}^{k} \overline{C F}^{k},
$$

where $\overline{C F}^{k}=\left[\rho \bar{H}^{T} \bar{u}^{k}-\log \left(\bar{x}^{k}\right)+C\right]^{q(k)}, \bar{u}^{k}=\left(\bar{y}-\bar{H} \bar{x}^{k}\right) /$ $\bar{H} \bar{x}^{k}$. Index is used with exponent, $q$, in order to show the result for AAMAPE in which exponent varies with iteration. For any $\bar{x}^{k_{1}}, \bar{x}^{k_{2}}$ in $R^{n}$ we get the following relation:

$$
\begin{aligned}
& \bar{x}^{k_{1}+1}=A\left(k_{1}\right) \bar{x}^{k_{1}} \overline{C F}^{k_{1}}, \\
& \bar{x}^{k_{2}+1}=A\left(k_{2}\right) \bar{x}^{k_{2}} \overline{C F}^{k_{2}} .
\end{aligned}
$$

Upon using (23), we get Euclidian distance between $\bar{x}^{k_{1}+1}$, $\bar{x}^{k_{2}+1}$ as follows:

$$
\begin{aligned}
d\left(\bar{x}^{k_{2}+1}, \bar{x}^{k_{1}+1}\right) & =\sum_{j}\left(x_{j}^{k_{2}+1}-x_{j}^{k_{1}+1}\right)^{2} \\
& =\sum_{j}\left(p_{j}^{k_{2}} x_{j}^{k_{2}}-p_{j}^{k_{1}} x_{j}^{k_{1}}\right)^{2},
\end{aligned}
$$

where $p_{j}^{k}=A(k) \overline{C F}_{j}^{k}$. Putting value of $A(k)$ from (17) and $\sum_{j} x_{j}^{k}=N$, we get following relation for $p_{j}^{k}$ :

$$
p_{j}^{k}=\frac{\left(\sum_{j} x_{j}^{k}\right)\left\{\rho\left(\bar{H}^{T} \bar{u}^{k}\right)_{j}-\log x_{j}^{k}+C\right\}^{q(j)}}{\sum_{j} x_{j}^{k}\left\{\rho\left(\bar{H}^{T} \bar{u}^{k}\right)_{j}-\log x_{j}^{k}+C\right\}^{q(j)}} .
$$

Since $C>\left(\rho+\log \left(\bar{x}^{k}\right)\right),\left|\bar{H}^{T} \bar{u}^{k}\right| \ll 1$, and $\bar{x}^{k}>0$, therefore $p_{j}^{k}>0$. It is also evident from (25) that $p_{j}^{k} \leq 1$. Replacing $p_{j}^{k}$ in (24) by minimum of all $p_{j}^{k}$, we get following inequality:

$$
\begin{aligned}
d\left(\bar{x}^{k_{2}+1}, \bar{x}^{k_{1}+1}\right) & \leq p^{2} \sum_{j}\left(x_{j}^{k_{2}}-x_{j}^{k_{1}}\right)^{2} \\
& =p^{2} d\left(\bar{x}^{k_{2}}, \bar{x}^{k_{1}}\right),
\end{aligned}
$$

where $p=\min \left\{p_{1}^{k_{1}}, p_{2}^{k_{1}}, \ldots, p_{n}^{k_{1}}, p_{1}^{k_{2}}, p_{2}^{k_{2}}, \ldots, p_{n}^{k_{2}}\right\}$ is lying between 0 and 1. Thus, for any $\bar{x}^{k_{1}}, \bar{x}^{k_{2}}$ in $R^{n}$ there exists a constant $0<c^{\prime}\left(=p^{2}\right)<1$ such that inequality (21) holds; and therefore contraction mapping property is satisfied for (15).

\subsection{Implementation and computational considerations}

In implementation of MAPE and AAMAPE, we use the shift-invariant property of the PSF. For linear shift-invariant system, convolution in spatial domain is equivalent to pointwise multiplication in Fourier domain [31]. In the MAPE and AAMAPE, evaluation of the array $\bar{H}^{T}\left(\bar{y} / \bar{H} \bar{x}^{k}\right)$ involves the heaviest computation in each iteration. This has been accomplished using Fast Fourier Transform (FFT) $\hat{h}(\xi, \eta), \hat{x}^{k}(\xi, \eta)$ of the PSF, $h$, and the image corresponding to $\bar{x}^{k}$, in four steps as follows: (1) form $\bar{H} \bar{x}^{k}$ by taking inverse FFT of the product $\hat{h}(\xi, \eta) \hat{x}^{k}(\xi, \eta)$; (2) replace all elements less than 1 by 1 in $\bar{H} \bar{x}^{k}$, and form the ratio, $\bar{y} / \bar{H} \bar{x}^{k}$, in the spatial domain; (3) compute the FFT of the result obtained in step 2 and multiply it by complex conjugate of $\hat{h}(\xi, \eta) ;(4)$ take the inverse FFT of the result of step 3 and replace all negative entries by zero.

The FFT is the heaviest computation in each iteration of the MAPE and AAMAPE method. Thus the overall computational complexity of these methods is $O(M N \log M N)$.

\section{SUPERRESOLUTION AND NOISE AMPLIFICATION IN AAMAPE}

\subsection{Superresolution}

It is often mentioned that the nonlinear methods have superresolution capability, restoring the frequency beyond the diffraction limit, without any rigorous mathematical support. In spite of highly nonlinear nature of AAMAPE method, we explain its superresolution characteristic qualitatively by using simplified form of (15) given as

$$
\bar{x}^{k+1}=A K^{q} \bar{x}^{k}+q \rho A K^{q-1} \bar{x}^{k} \bar{H}^{T} \bar{u}^{k},
$$

where $\bar{u}^{k}=\left(\bar{y}-\bar{H} \bar{x}^{k}\right) / \bar{H} \bar{x}^{k}$, and $K=C-\log \left(\bar{x}^{k}\right)$. Derivation of (27) is given in Appendix A. An equivalent expression of (27) in Fourier domain is obtained by using convolution, correlation theorem as [31]

$$
X^{k+1}(\bar{f})=A K^{q} X^{k}(\bar{f})+\frac{q \rho A K^{q-1}}{M N} X^{k}(\bar{f}) \otimes H^{*}(\bar{f}) U^{k}(\bar{f}),
$$

where superscript $*$ denotes the conjugate transpose of a matrix; and $X^{k+1}, X^{k}$, and $U^{k}$ are discrete Fourier transforms of size $M \times N$ corresponding to the variable in lower case letters; $\bar{f}$ is 2-D frequency index. $H$ is the Fourier transform of PSF, $h$, and it is known as optical transfer function (OTF). The OTF is band limited, say, its upper cutoff frequency is $f_{C}$; that is, $H(\bar{f})=0$ for $|\bar{f}|>f_{C}$. In order to make explanation of superresolution easy, we rewrite (28) as follows:

$$
\begin{aligned}
X^{k+1}(\bar{f})= & A K^{q} X^{k}(\bar{f}) \\
& +\frac{q \rho A K^{q-1}}{M N} \sum_{v} X^{k}(\bar{f}-v) H^{*}(v) U^{k}(v) .
\end{aligned}
$$

At any iteration, the product $H^{*} U^{k}$ in (29) is also band limited and has the frequency support at most as that of $H$. 
TABLE 1: Blurring PSF, BSNR, and SNR.

\begin{tabular}{lcccc}
\hline Experiment & Image & $\begin{array}{c}\text { Blurring } \\
\text { PSF }\end{array}$ & $\begin{array}{c}\text { BSNR } \\
{[\mathrm{dB}]}\end{array}$ & $\begin{array}{c}\text { SNR } \\
{[\mathrm{dB}]}\end{array}$ \\
\hline Exp.1 & Cameraman & $5 \times 5$ Box-car & 40 & 17.35 \\
Exp.2 & Lenna & $5 \times 5$ Box-car & 32 & 20.27 \\
Exp.3 & Anuska & $5 \times 5$ Gauss, scale $=3$ & 38.60 & 22.95 \\
\hline
\end{tabular}

Due to multiplication of $H^{*} U^{k}$ by $X^{k}$ and summation over all available frequency indexes, second term of (29) is never zero. Indeed, the inband frequency components of $X^{k}$ are spread out of the band. Thus, the restored image spectrum, $X^{k+1}(\bar{f})$, has frequencies beyond the cutoff frequency, $f_{C}$. It is important to note that the observation, $\bar{y}$, is noisy and noise has frequency component at high frequencies. Thus, the restored frequencies are contaminated and spurious high frequencies are also present in restored image. How to ensure the fidelity of the restored frequencies and rejection of spurious high frequencies without knowing the frequency present in the true data, $\bar{x}$, is challenging and an open problem.

\subsection{Noise amplification}

In this section, signal dependent noise characteristic of noise amplification has been investigated qualitatively. It is worth noting that the complete recovery of frequencies present in true image from the observed image requires large number of iterations. But due to noisy observation, noise also amplifies as iteration increases. Hence, restored image may become unacceptably noisy and unreliable at large number of iterations. Noise in $(k+1)$ th iteration is estimated by finding the correlation of the deviation of restored spectra $X^{k+1}(\bar{f})$ form its expected value $E\left[X^{k+1}(\bar{f})\right]$. This correlation is the measure of noise and is given as follows:

$$
\begin{aligned}
& \left.\mu_{X}^{k+1}\left(\bar{f}, \bar{f}^{\prime}\right)\right\} \\
& \quad=E\left[\left\{X^{k+1}(\bar{f})-E\left[X^{k+1}(\bar{f})\right]\left\{X^{k+1}(\bar{f})-E\left[X^{k+1}(\bar{f})\right]\right\}^{*}\right],\right.
\end{aligned}
$$

where $*$ denotes the complex conjugate. In order to get simple and compact expression for amplified noise (30), we assume that the correlation at two different spatial frequencies is independent, that is, vanishing correlation at two different spatial frequencies. Substituting $X^{k+1}$ from (29) into (30) and using this assumption we get following relation:

$$
\begin{aligned}
N_{X}^{k+1}(\bar{f})-N_{X}^{k}(\bar{f})= & \left(\alpha^{2}-1\right) N_{X}^{k}(\bar{f}) \\
& +\beta^{2} \sum_{v}|H(v)|^{2}\left|U^{k}(v)\right|^{2} N_{X}^{k}(\bar{f}-v) \\
& +2 \alpha \beta \sum_{v} \operatorname{Re}\left(H^{*}(v) U^{k}(v)\right) E\left(\left|X^{k}(\bar{f})\right|^{2}\right) \\
& -2 \alpha \beta \sum_{v} \operatorname{Re}\left(H^{*}(v) U^{k}(v)\right)\left|E\left(X^{k}(\bar{f})\right)\right|^{2},
\end{aligned}
$$

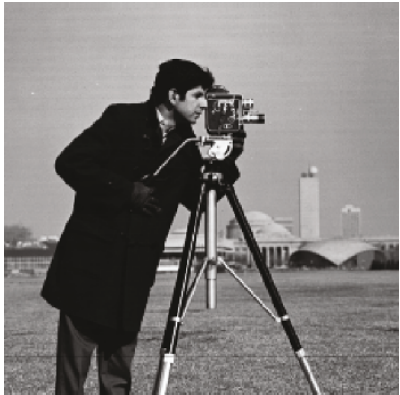

(a)

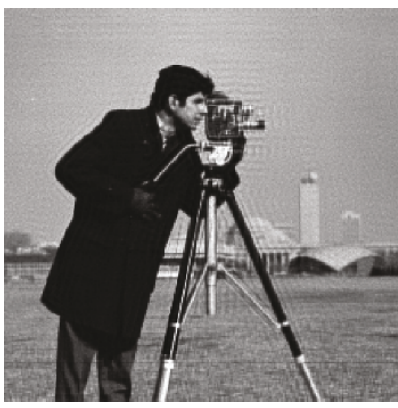

(c)

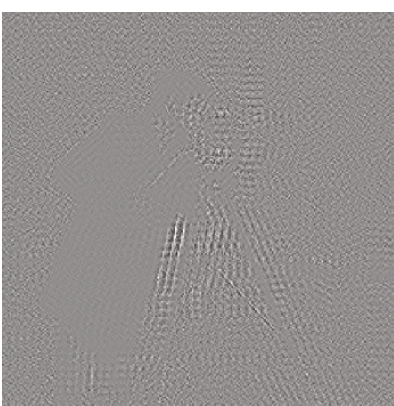

(e)

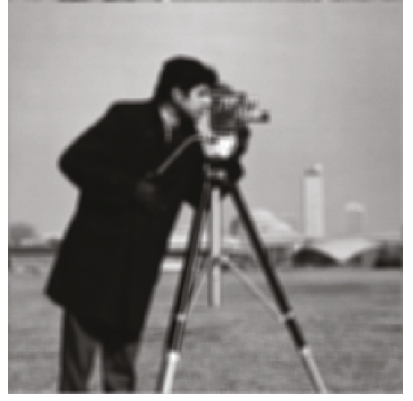

(b)

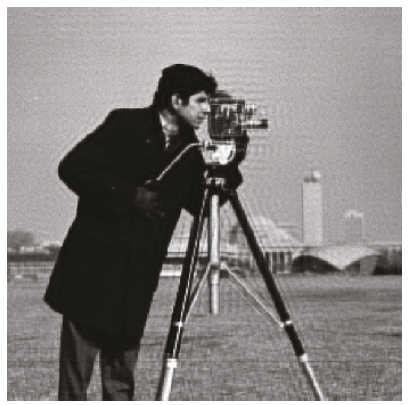

(d)

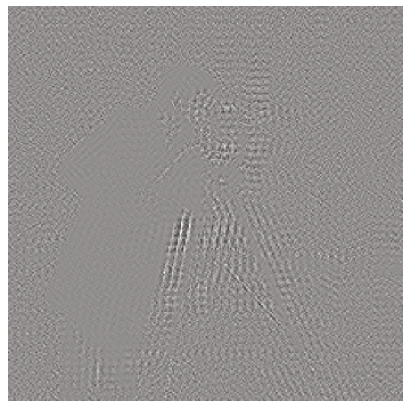

(f)
Figure 1: "Cameraman" image (a) original image, (b) noisy and blurred image; PSF $5 \times 5$ Box-Car, BSNR $=40 \mathrm{~dB}$, (c) restored image by MAPE corresponding to maximum SNR (348 iterations), (d) restored image by AAMAPE correspondsing to maximum SNR (201 iterations), (e) residual corresponding (c), and (f) residual corresponding $(\mathrm{d})$.

where, $\alpha=A K^{q}, \beta=q \rho A K^{q-1} / M N$ and $N_{X}^{k}(\bar{f})=\mu_{X}^{k}(\bar{f}, \bar{f})$ represents the noise in $X^{k}$ at frequency $\bar{f}$. Derivation of (31) is given in Appendix B. From third and fourth term of (31), it is clear that in AAMPAE amplified noise is signal dependent. Moreover, noise from one iteration to next is cumulative. Thus, increasing number of iterations does not guarantee that the restored quality of image will become acceptable. We can find total amplified noise by summing (31) over all MN frequencies, provided that the statistics of the restored spatial frequency is known at each iteration. But in practice, there is no way to estimate statistics of spatial frequency- $E\left(X^{k}(\bar{f})\right)$, $E\left(\left|X^{k}(\bar{f})\right|^{2}\right)$-at each iteration. Moreover, due to intricate relation between spatial frequencies it is almost impossible 


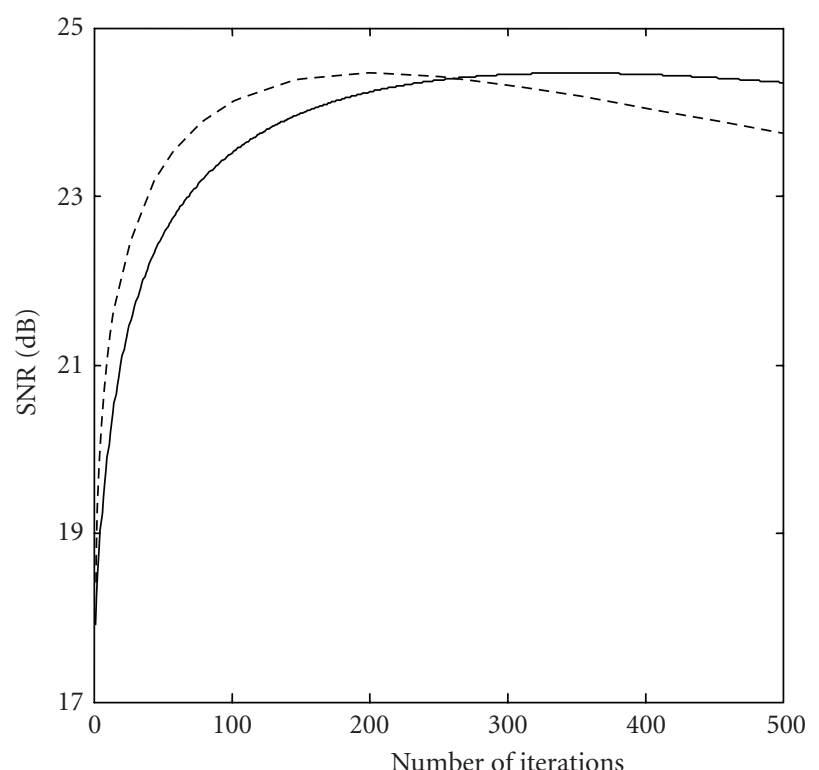

(a)

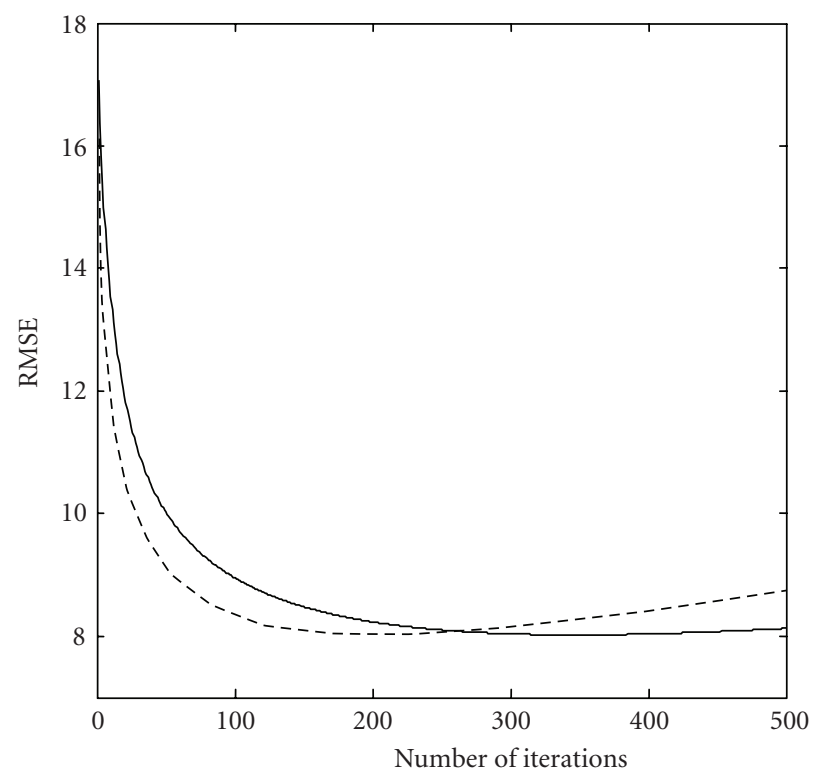

(b)

FIGURE 2: "Cameraman" image (a) SNR of the MAPE (solid line) and AAMAPE (dotted line). (b) RMSE of the MAPE (solid line) and AAMAPE (dotted line).

to give reliable and exact noise amplification formula. In derivation of (31), we use the assumption that one spatial frequency is independent from the other, that is, correlation term at two different spatial frequencies is zero. Thus, Monte Carlo simulations are the only realistic way to assess the amplified noise. Equation (31) is for understanding the wellknown fact that the amplified noise is signal dependent in this nonlinear method.

\section{EXPERIMENTAL RESULTS AND DISCUSSIONS}

In this section, we describe three experiments demonstrating the performance of the AAMAPE method in comparison

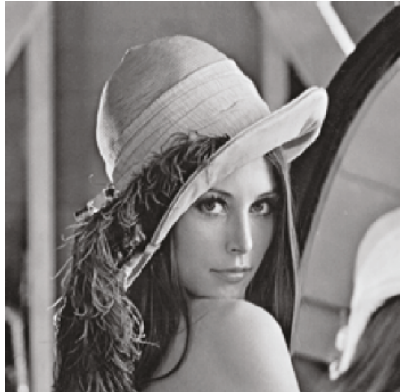

(a)

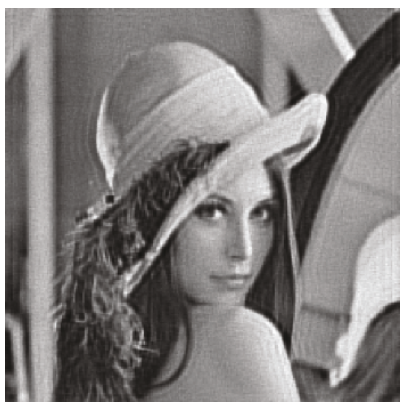

(c)

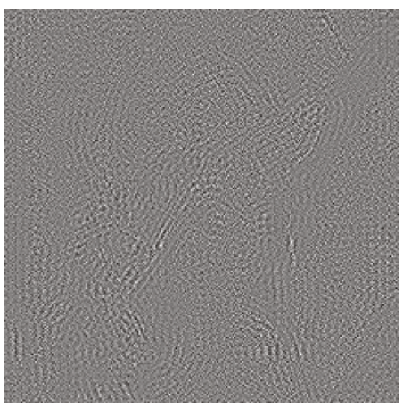

(e)

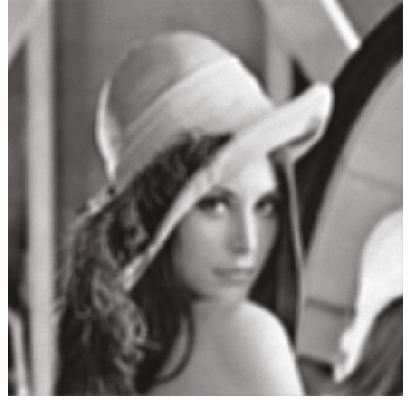

(b)

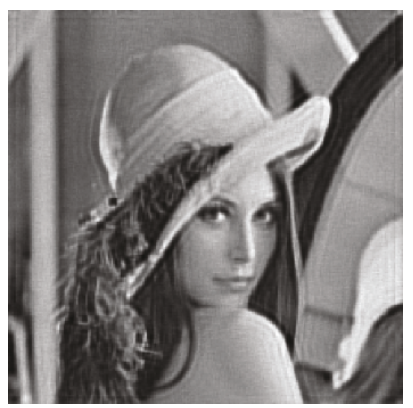

(d)

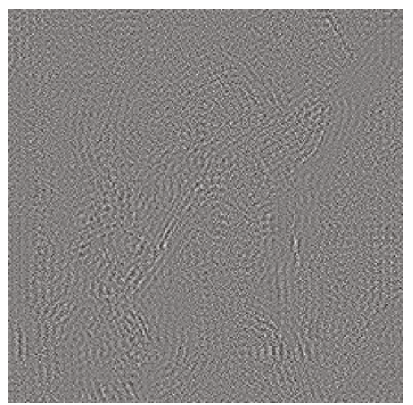

(f)
FIGURE 3: "Lenna" image (a) original image, (b) noisy and blurred image; PSF $5 \times 5$ Box-Car, BSNR $=32 \mathrm{~dB}$, (c) restored image by MAPE corresponding to maximum SNR (79 iterations), (d) restored image by AAMAPE corresponding to maximum SNR (44 iterations), (e) residual corresponding (c), and (f) Residual corresponding $(\mathrm{d})$.

with MAPE method. Test images used in these experiments are Cameraman (Experiment 1), Lenna (Experiment 2), and Anuska (Experiment 3); all are $256 \times 256$, 8-bit gray scale image. The corrupting noise is of Poisson type for both experiments. Table 1 displays the blurring PSF, BSNR, and SNR for all three experiments. The level of noise in the observed image is characterized in decibels by blurred SNR (BSNR) and defined as

$$
\begin{aligned}
\mathrm{BSNR} & =10 \log _{10}\left[\frac{\sum\left(\bar{H} \bar{x}-(1 / M N) \sum \bar{H} \bar{x}\right)^{2}}{\sigma^{2} M N}\right] \\
& \approx 10 \log _{10}\left[\frac{\sum\left(\bar{H} \bar{x}-(1 / M N) \sum \bar{H} \bar{x}\right)^{2}}{\sum(\bar{y}-H \bar{x})^{2}}\right],
\end{aligned}
$$


TABLE 2: SNR, iterations, and time for MAPE, AAMAPE, WaveGsm_TI [32], ForWaRD [33], and RI [34].

\begin{tabular}{|c|c|c|c|c|c|c|c|c|c|}
\hline \multirow{2}{*}{ Method } & \multicolumn{3}{|c|}{ SNR [dB] } & \multicolumn{3}{|c|}{ No. of iterations } & \multicolumn{3}{|c|}{ Time [sec.] } \\
\hline & Exp.1 & Exp.2 & Exp.3 & Exp.1 & Exp.2 & Exp.3 & Exp.1 & Exp.2 & Exp.3 \\
\hline MAPE & 24.46 & 24.54 & 28.63 & 348 & 79 & 124 & 39.1 & 9.2 & 14.6 \\
\hline AAMAPE & 24.46 & 24.54 & 28.65 & 201 & 44 & 71 & 22.5 & 5.0 & 7.8 \\
\hline WaveGSM_TI & 21.63 & 21.81 & 27.84 & 504 & 164 & 105 & 2349.4 & 772.57 & 601.5 \\
\hline ForWaRD & 25.17 & 25.64 & 26.58 & -- & - & - & -- & -- & - \\
\hline RI & 25.50 & 25.74 & 27.94 & - & -- & -- & 20.0 & - & -- \\
\hline
\end{tabular}

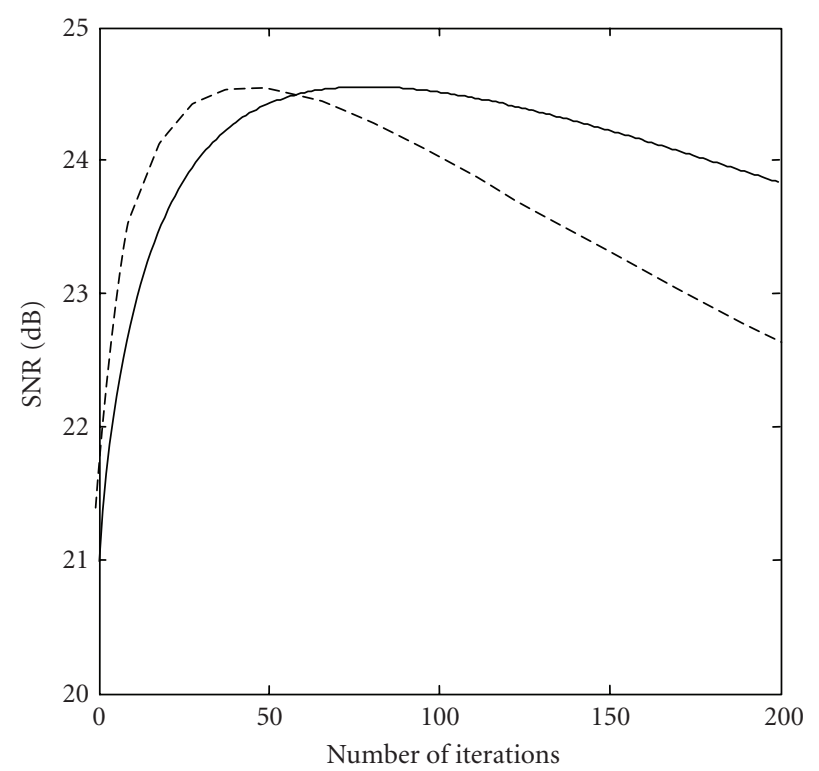

(a)

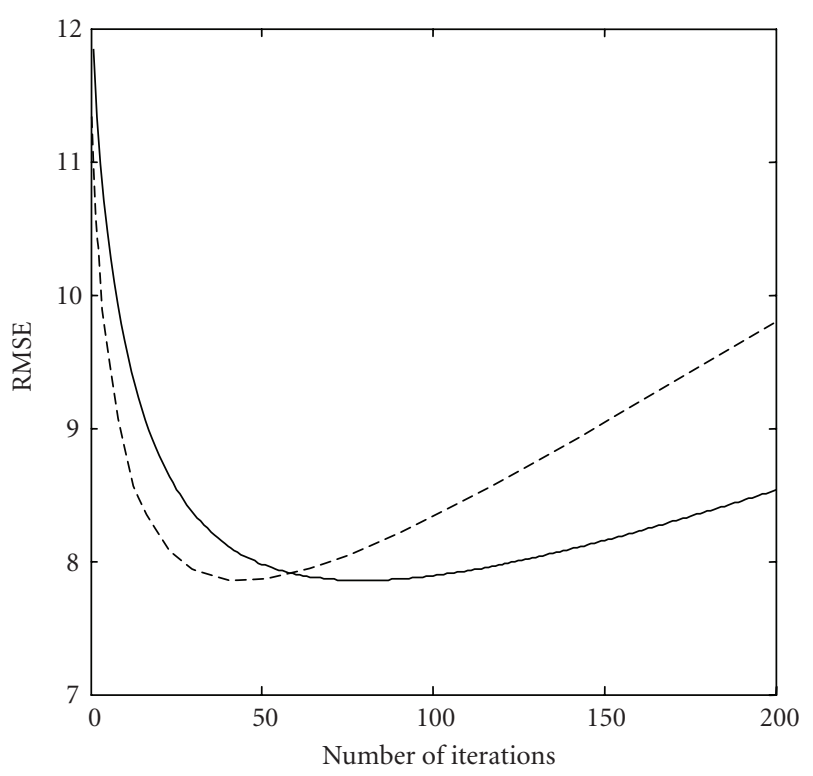

(b)

FIGURE 4: "Lenna" image (a) SNR of the MAPE (solid line) and AAMAPE (dotted line). (b) RMSE of the MAPE (solid line) and AAMAPE (dotted line). where $\sigma$ is the standard deviation of noise. The following standard imaging performance criterions are used for comparison of AAMAPE method and MAPE method:

$$
\begin{aligned}
& \mathrm{RMSE}=\sqrt{\left(\frac{1}{M N}\right) \sum\left(\bar{x}-\bar{x}^{k}\right)^{2}}, \\
& \mathrm{SNR}=10 \log _{10}\left(\frac{\sum|\bar{x}|^{2}}{\sum\left|\bar{x}-\bar{x}^{k}\right|^{2}}\right) .
\end{aligned}
$$

Most of these criteria actually define the accuracy of approximation of the image intensity function. There is no one-to-one link between the image quality and the above criteria. Another criterion based on residual, $\bar{r}=\bar{y}-\bar{H} \bar{x}^{k}$, is discussed in [14]. The deblurred image, $\bar{x}^{k}$, is acceptable, if the residual are consistent with statistical distribution of noise in observed image $\bar{y}$. If any systematic structure in residual is observed, or if the residual distribution is inconsistent with noise statistics of $\bar{y}$, there is something wrong with the deblurred image. In most of the deblurring method, particularly in linear methods, there is tradeoff between residual and artifacts appearing in the deblurred image [14]. Excellent residual does not guarantee that the deblurred image has less or free from artifact. Thus, a visual inspection, which of course is quite subjective, continues to be the most important final performance criterion.

Figures 1(c)-1(d), Figures 3(c)-3(d), and Figures 5(c)$5(\mathrm{~d})$ show the restored images, corresponding to the maximum SNR, of Experiments one, two, and three. It is clear from these figures that the AAMAPE gives almost same visual results in less number of iterations than MAPE method for all experiments. Figure 2, Figure 4, and Figure 6 show the variations of SNR, RMSE versus iterations for experiments. It is observed that the AAMAPE has faster increase in SNR and faster decrease in RMSE in comparison to that of MAPE method, for all three experiments. It is clear from Figures 1(e)-1(f), Figures 3(e)-3(f), and Figures 5(e)-5(f), that the AAMAPE gives same residual as MAPE in lesser number of iteration. Hence, the performance of the proposed AAMAPE method is consistently better than the MAPE method. In Figures 7(a)-7(c), it can be seen that the exponent $q$ has value near three at the start of the iteration and approaching to one as iterations increase. Thus, AAMAPE method prefers speed at the beginning stage of iteration, and stability at later stages. It can be observed in Figures 2, 4, and 6 that the SNR is increasing and the RMSE is decreasing up to certain number of iteration, and after that SNR decreases 


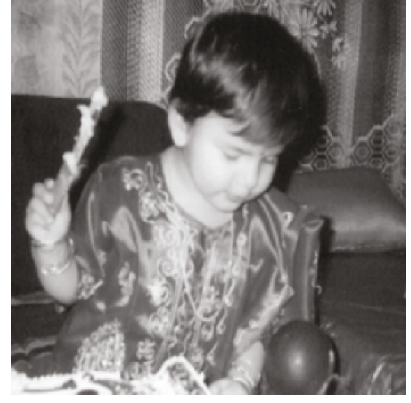

(a)

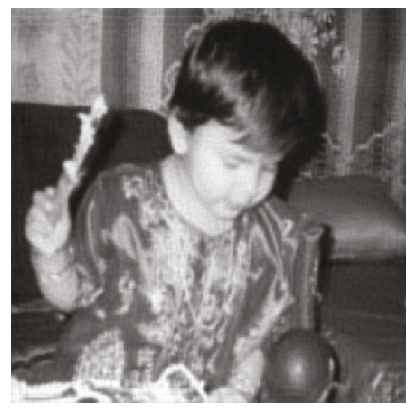

(c)

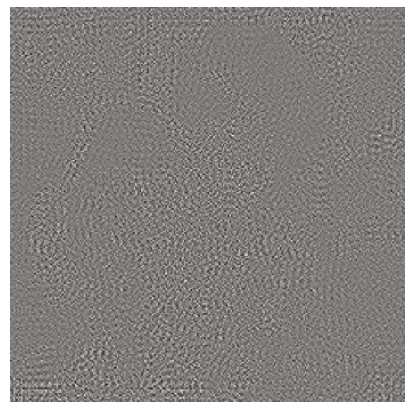

(e)

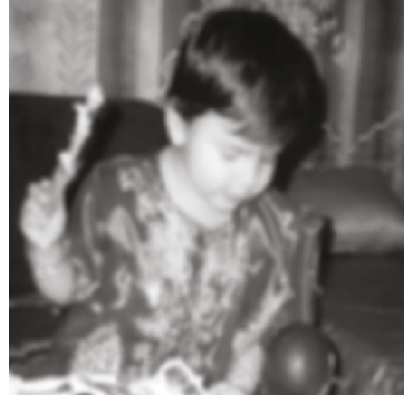

(b)

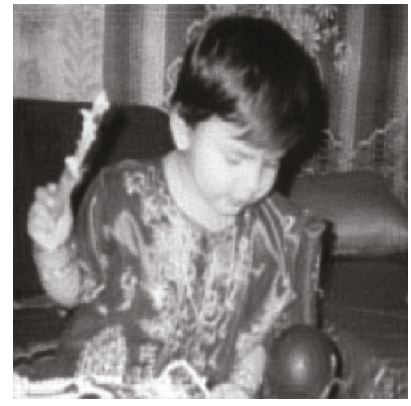

(d)

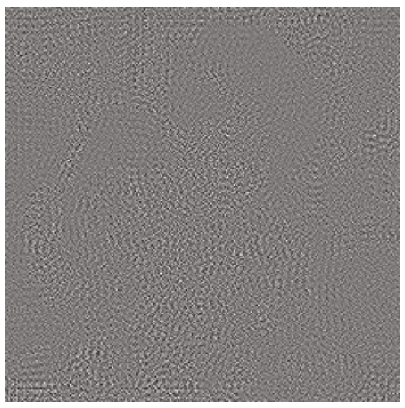

(f)
Figure 5: "Anuska" image (a) original image, (b) noisy and blurred image; PSF $5 \times 5$ Gauss, BSNR $=38.6 \mathrm{~dB}$, (c) restored image by MAPE corresponding to maximum SNR (124 iterations), (d) restored image by AAMAPE corresponding to maximum SNR (71 iterations), (e) residual corresponding (c), and (f) residual corresponding $(\mathrm{d})$.

and RMSE increases. This is due to the fact that the noise amplification is signal dependent, as discussed in Section 3. Thus, increasing number of iterations does not necessarily improve the quality of restored image. In order to terminate the iterations corresponding to the best result, some stopping criterion must be used [14, 35].

Table 2 shows the SNR, number of iterations, and computation time of the MAPE, proposed AAMAPE, WaveGSM_TI [32], ForWaRD [33], and RI [34] for Experiments 1-3. Matlab implementation of ForWaRD is available at http://www.dsp.rice.edu/software/, and RI at http:// www.cs.tut.fi/ lasip/ $\#$ re software.

The proposed AAMAPE method gives same SNR as MAPE in less iteration and less computation time. The performance of AAMAPE is better-higher SNR, less itera-

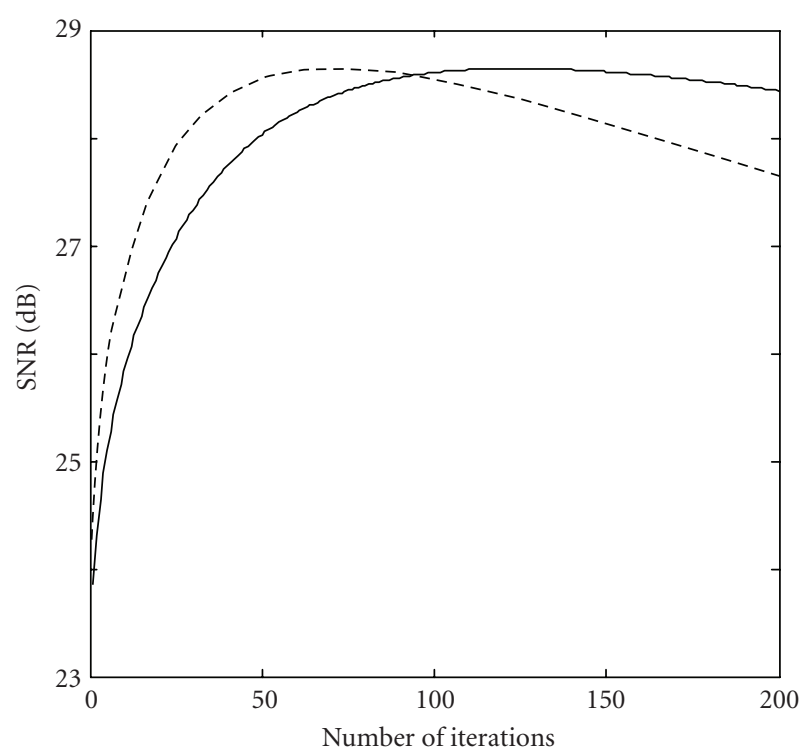

(a)

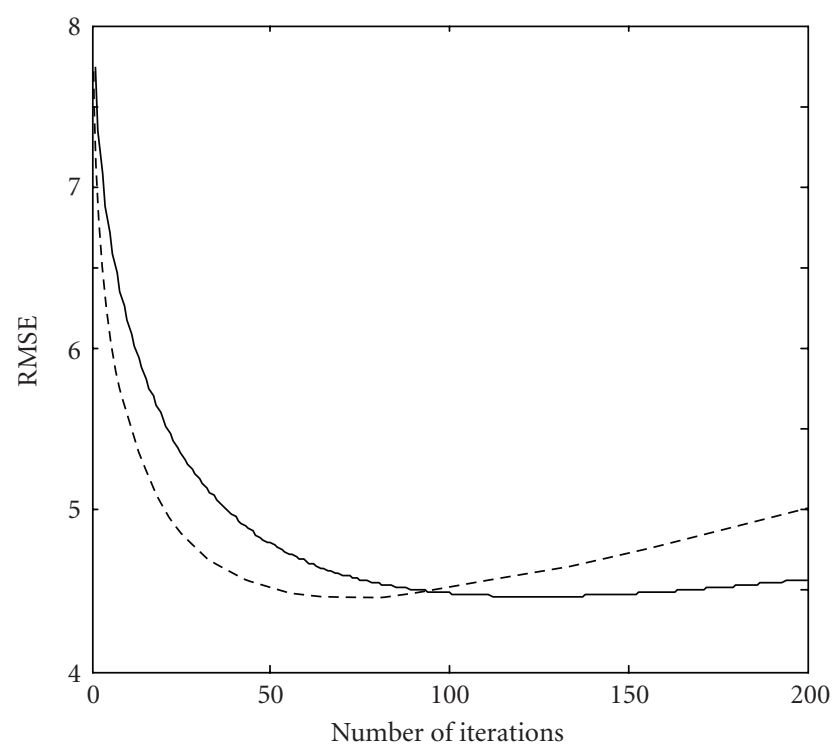

(b)

FIgure 6: "Anuska" image (a) SNR of the MAPE (solid line) and AAMAPE (dotted line). (b) RMSE of the MAPE (solid line) and AAMAPE (dotted line).

tion and less computation time-than the iterative method WaveGSM_TI. In Experiments 1 and 2, the SNR achieved in AAMAPE is less than ForWaRD and RI $(\approx 1 \mathrm{~dB})$. This is due to the fact that in the ForWaRD and the RI, deblurring is performed followed by denoising. We achieved the SNR 26.0 dB, 26.20 dB, and 29.3 dB, for Experiments 1, 2, and 3 , respectively; when the result obtained form AAMAPE is denoised by Wavelet-domain Wiener Filter (WWF) [36]. Hence, our proposed AAMAPE method with WWF yields higher SNR in comparison to the state-of-the-art methods ForWaRD and RI. Moreover, we performed experiments with many images of different types (optical, CT, MRI, 


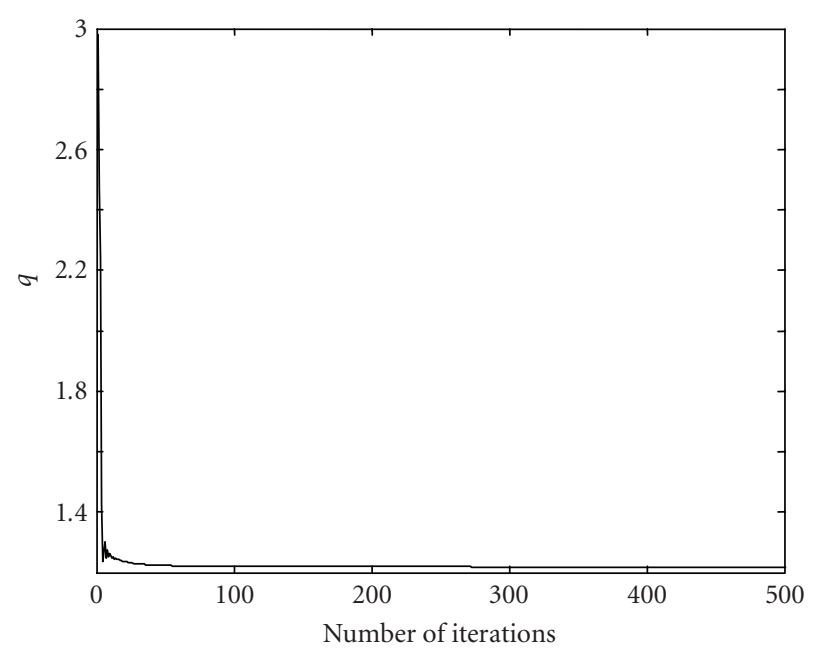

(a)

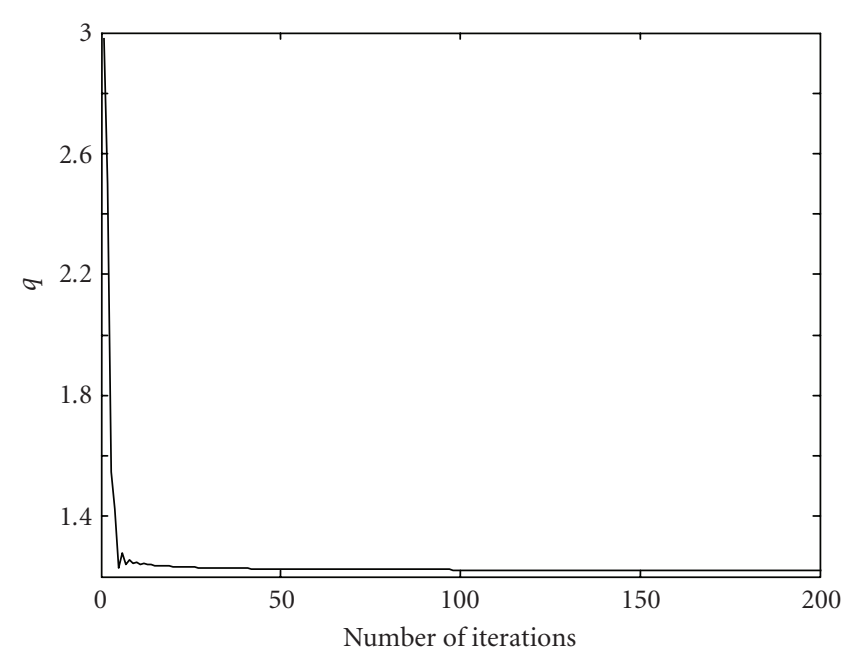

(b)

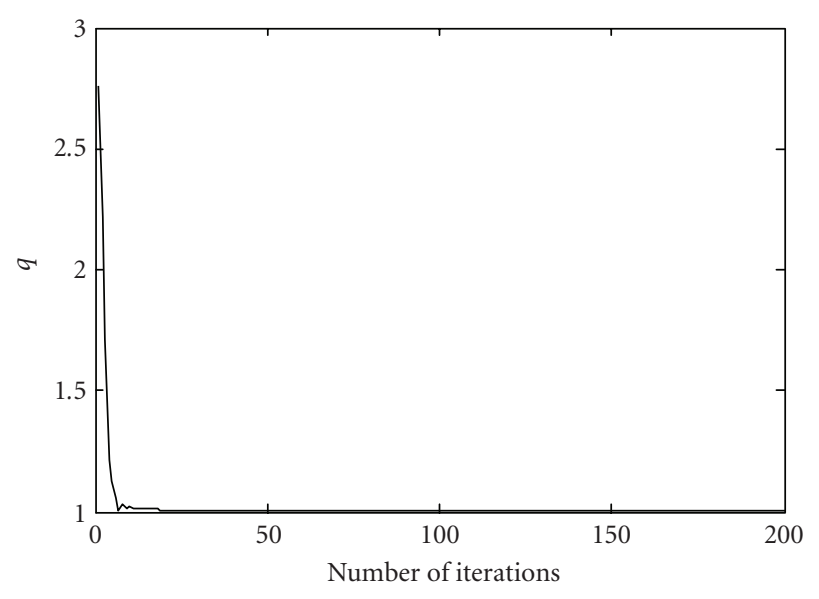

(c)

FIgURE 7: Iteration versus $q$ (a) Cameraman image, (b) Lenna image, and (c) Anuska image.

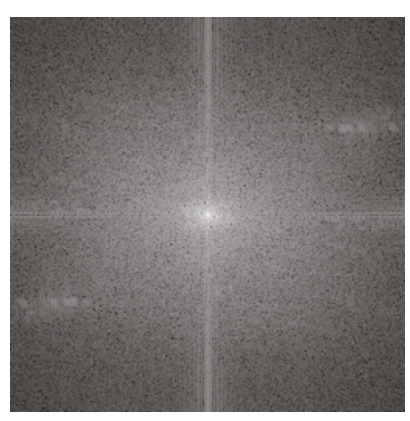

(a)

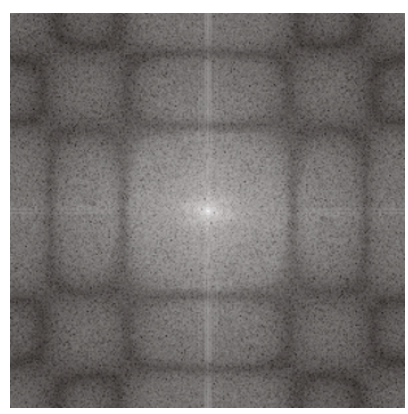

(c)

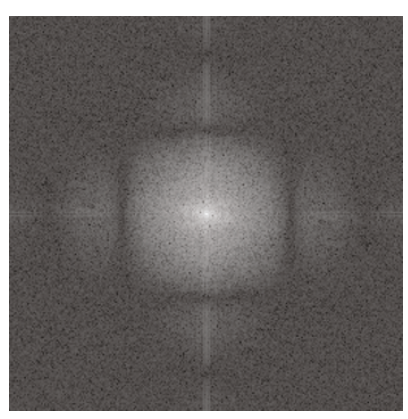

(b)

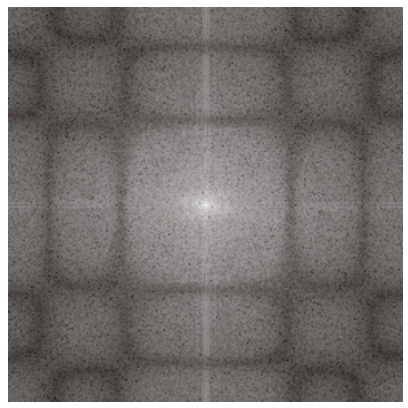

(d)
Figure 8: Spectra of images from Figure 5. All spectra are range compressed with $\log _{10}\left(1+|\cdot|^{2}\right)$, (a) original image in Figure 5(a), (b) blurred and noisy image Figure 5(b), (c) Figure 5(c), and (d) Figure 5(d).

ultrasound), with different blurs and noise levels; we found that the proposed AAMAPE performs better than the stateof-the-arts methods [32-34].

In order to illustrate the superresolution capability of the MAPE and AAMAPE, we present the spectra of the original, blurred, and restored images in Figure 8 for the third experiment. It is evident that the restored spectra, as given in Figures $8(\mathrm{c})-8(\mathrm{~d})$, have frequency component that are not present in observed spectra as in Figure 8(b). But restored spectra are not identical to that of original image spectra as shown in Figure 8(a). In principle, an infinite number of iteration are required to recover the true spectra from the observed spectra using any nonlinear method. But due to noisy observation, noise also gets amplified as the number of iteration increases and the quality of restored image degrades.

\section{CONCLUSIONS}

In this paper, we proposed AAMAPE method for image deblurring. The AAMAPE method uses a multiplicative correction term which has been calculated using an exponent on the correction factor. The proposed empirical technique computes the exponent adaptively in each iteration using first-order derivative of the restored image in previous two iterations. With this exponent, the AAMAPE method emphasized speed and stability, respectively, at the early and late stages of iterations. The experimental investigations 
suggest that AAMAPE method give better results in terms of low RMSE and high SNR, even when 44\% lesser iterations than MAPE method. This adaptive method has simple form and very easy to implement. Moreover, computations required per iteration in AAMAPE are almost the same as those in MAPE. AAMAPE yields better result, in terms of SNR, than the recently published state-of-the-art methods. The noise amplification and the superresolution capability of AAMAPE method are not evident due to extremely intricate restoration process. We explained the superresolution property of the proposed accelerated method analytically and verified it experimentally. For interpreting the noise amplification of the proposed method, we have also performed an analytical analysis, which confirms its signal dependent nature of amplified noise.

In AAMAPE, we assumed that the PSF is known and is shift-invariant. However, in many cases, the PSF is unknown. In such blind deblurring problems, the PSF and true image must be estimated simultaneously from noisy and blurred observation. Extension of AAMAPE for shift-variant PSF and blind deblurring is subject of future research.

\section{APPENDICES}

\section{A. DERIVATION OF (27)}

The term in square bracket of (15) is expressed as follows:

$$
\begin{aligned}
& \rho \bar{H}^{T}\left(\frac{\bar{y}}{\bar{H} \bar{x}^{k}}\right)-\rho-\log \left(\bar{x}^{k}\right)+C \\
& \quad=\rho\left[\bar{H}^{T}\left(\frac{\bar{y}}{\bar{H} \bar{x}^{k}}-\overline{1}+\overline{1}\right)-\rho-\log \left(\bar{x}^{k}\right)+C\right] \\
& \quad=\rho \bar{H}^{T}\left(\frac{\bar{y}-\bar{H} \bar{x}^{k}}{\bar{H} \bar{x}^{k}}\right)-\log \left(\bar{x}^{k}\right)+C \\
& \quad=\rho \bar{H}^{T} \bar{u}^{k}-\log \left(\bar{x}^{k}\right)+C,
\end{aligned}
$$

where $\bar{u}^{k}$ is relative fitting error and it is given as follows:

$$
\bar{u}^{k}=\frac{\bar{y}-\bar{H} \bar{x}^{k}}{\bar{H} \bar{x}^{k}} \text {. }
$$

Raising power $q$ both sides of (A.1), we get

$$
\left[\rho \bar{H}^{T}\left(\frac{\bar{y}}{\bar{H} \bar{x}^{k}}\right)-\rho-\log \left(\bar{x}^{k}\right)+C\right]^{q}=K^{q}\left[1+\frac{\rho}{K} \bar{H}^{T} \bar{u}^{k}\right]^{q},
$$

where $K=C-\log \left(\bar{x}^{k}\right)$. It is observed that $\left|\bar{H}^{T} \bar{u}^{k}\right| \ll 1$, for sufficiently large $k$. Moreover, by the Riemann-Lebesgue lemma it is possible to show that the sum $\bar{H}^{T} \bar{u}^{k}$ has value very close to zero [4]. It is obvious that $\rho / K<1$, and hence, $\left|(\rho / K) \bar{H}^{T} \bar{u}^{k}\right| \ll 1$. Expanding square bracket in left-hand side of (A.3) using Taylor series expansion:

$$
\begin{aligned}
K^{q}[1 & \left.+\frac{\rho}{K} \bar{H}^{T} \bar{u}^{k}\right]^{q} \\
& =K^{q}\left[1+q \frac{\rho}{K} \bar{H}^{T} \bar{u}^{k}+\frac{q(q-1)}{2 !}\left(\frac{\rho}{K} \bar{H}^{T} \bar{u}^{k}\right)^{2}+\cdots\right] \\
& =K^{q}+q \rho K^{q-1} \bar{H}^{T} \bar{u}^{k}+\frac{q(q-1)}{2 !} K^{q}\left(\frac{\rho}{K} \bar{H}^{T} \bar{u}^{k}\right)^{2}+\cdots
\end{aligned}
$$

In both experiments we use $\rho=10000, C=11000$, and found that at higher iteration most of the values of $\left|\bar{H}^{T} \bar{u}^{k}\right|$ are in between $10^{-10}$ and $10^{-8}$. Moreover, we observed that the $\left|\bar{H}^{T} \bar{u}^{k}\right|$ fall below $10^{-2}$ just in four iterations. In order to calculate maximum possible value of third term of (A.4), at higher iteration, we take image intensity value 255 and $q=3$. From these we found that third term of (A.4) is between $10^{-8}$ and $10^{-4}$. Thus, the third and higher order term in (A.4) is very small. Retaining only first-order term we arrive on following relation:

$$
\left[\rho \bar{H}^{T}\left(\frac{\bar{y}}{H^{T} \bar{x}}\right)-\rho-\log \left(\bar{x}^{k}\right)+C\right]^{q}=K^{q}+q \rho K^{q-1} \bar{H}^{T} \bar{u}^{k} .
$$

Using (A.5) in (15) we get the following:

$$
\bar{x}^{k+1}=A K^{q} \bar{x}^{k}+q \rho A K^{q-1} \bar{x}^{k} \bar{H}^{T} \bar{u}^{k} .
$$

In order to validate this approximation, we perform the simulation for Experiment 1. The results are shown in Figure 9. From Figures 9(a)-9(b) and Figures 1(d), 1(f), we see that the simplified form, (A.6), gives almost same restored image and residual as AAMAPE. Moreover, from Figure 9(c), we see that RMSE variation of simplified form, (A.6), agrees with RMSE of AAMAPE.

\section{B. DERIVATION OF $(31)$}

In order to make mathematical step understandable, we rewrite (29) as follows:

$$
X^{k+1}(\bar{f})=\alpha X^{k}(\bar{f})+\beta Y^{k}(\bar{f}),
$$

where

$$
Y^{k}(\bar{f})=\sum_{v} X^{k}(\bar{f}-v) H^{*}(v) U^{k}(v)
$$

For estimating noise amplification during iteration, we use covariance analysis. By using covariance, we find the rule for evolution of spatial frequency from one iteration to next iteration. By using (30) and (B.1), covariance of $X^{k+1}$ for two different spatial frequencies is given as

$$
\begin{aligned}
\mu_{X}^{k+1}\left(\bar{f}, \bar{f}^{\prime}\right)= & \alpha^{2} \mu_{X}^{k}\left(\bar{f}, \bar{f}^{\prime}\right)+\beta^{2} \mu_{Y}^{k}\left(\bar{f}, \bar{f}^{\prime}\right) \\
& +\alpha \beta \mu_{X Y^{*}}^{k}\left(\bar{f}, \bar{f}^{\prime}\right)+\alpha \beta \mu_{X^{*} Y}^{k}\left(\bar{f}, \bar{f}^{\prime}\right),
\end{aligned}
$$




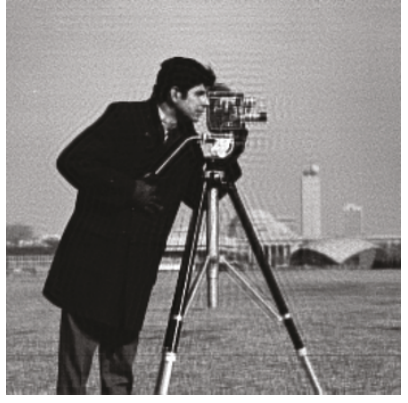

(a)

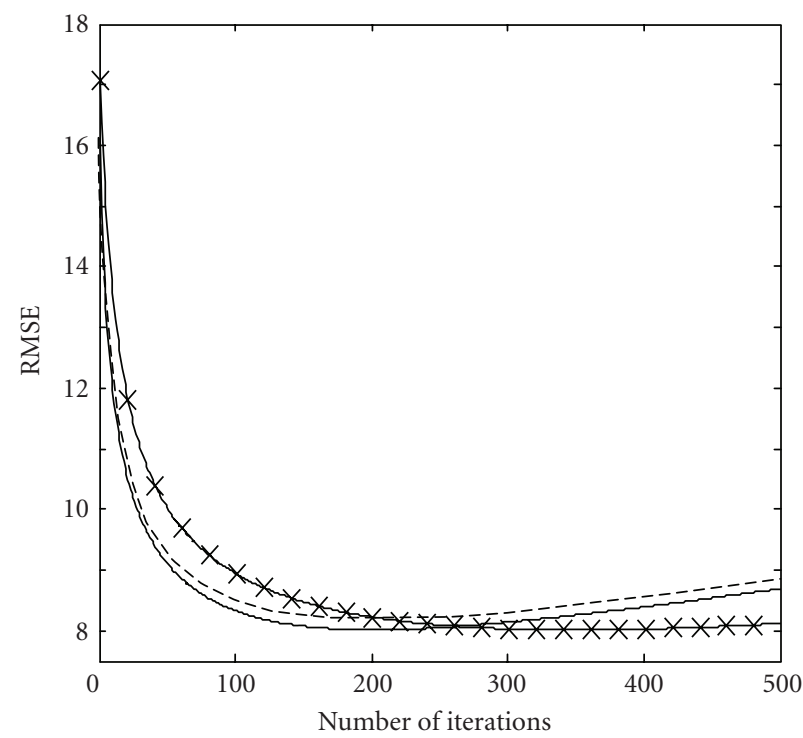

(c)

FIGURE 9: Result for Experiment 1 using (27): (a) restored image by simplified form (27) of AAMAPE (15) corresponding to maximum SNR (203 Iterations), (b) residual corresponding (a), and (c) RMSE of the MAPE (solid line with $x$ ), AAMAPE (solid line), and AAMAPE simplified form given by (27) (dotted line).

where

$$
\begin{aligned}
& \mu_{X Y^{*}}^{k}\left(\bar{f}, \bar{f}^{\prime}\right) \\
& \quad=E\left[\left\{X^{k}(\bar{f})-E\left[X^{k}(\bar{f})\right]\right\}\left\{Y^{k^{*}}\left(\bar{f}^{\prime}\right)-E\left[Y^{k^{*}}\left(\bar{f}^{\prime}\right)\right]\right\}\right] .
\end{aligned}
$$

Using (30) and (B.2) we get

$$
\begin{gathered}
\mu_{Y}^{k}\left(\bar{f}, \bar{f}^{\prime}\right)=\sum_{v} \sum_{v^{\prime}}\left\{H(v) H^{*}\left(v^{\prime}\right) U^{k}(v) U^{k^{*}}\left(v^{\prime}\right)\right. \\
\left.\times E\left[X^{k}(\bar{f}-v) X^{k^{*}}\left(\bar{f}^{\prime}-v^{\prime}\right)\right]\right\} \\
-\sum_{v} \sum_{v^{\prime}}\left\{H(v) H^{*}\left(v^{\prime}\right) U^{k}(v) U^{k^{*}}\left(v^{\prime}\right)\right. \\
\left.\times E\left[X^{k}(\bar{f}-v)\right] E\left[X^{k^{*}}\left(\bar{f}^{\prime}-v^{\prime}\right)\right]\right\}, \\
\mu_{Y}^{k}\left(\bar{f}, \bar{f}^{\prime}\right) \quad \\
=\sum_{v} \sum_{v^{\prime}}\left\{H(v) H^{*}(v) U^{k}(v) U^{k^{*}}\left(v^{\prime}\right) \mu_{X}^{k}\left(\bar{f}-v, \bar{f}^{\prime}-v\right)\right\} .
\end{gathered}
$$

Using (B.2) into (B.4) we get $\mu_{X Y^{*}}^{k}$

$$
\begin{aligned}
\mu_{X Y^{*}}^{k}\left(\bar{f}, \bar{f}^{\prime}\right) & \\
= & \sum_{v}\left\{H(v) U^{k^{*}}(v) E\left[X^{k}(\bar{f}) X^{k^{*}}\left(\bar{f}^{\prime}-v\right)\right]\right\} \\
& -\sum_{v}\left\{H(v) U^{k^{*}}(v) E\left[X^{k}(\bar{f})\right] E\left[X^{k^{*}}\left(\bar{f}^{\prime}-v\right)\right]\right\} .
\end{aligned}
$$

Similarly,

$$
\begin{aligned}
\mu_{X^{*} Y}^{k}\left(\bar{f}, \bar{f}^{\prime}\right) & \\
= & \sum_{v}\left\{H^{*}(v) U^{k}(v) E\left[X^{k^{*}}(\bar{f}) X^{k}\left(\bar{f}^{\prime}-v\right)\right]\right\} \\
& -\sum_{v}\left\{H^{*}(v) U^{k}(v) E\left[X^{k^{*}}(\bar{f})\right] E\left[X^{k}\left(\bar{f}^{\prime}-v\right)\right]\right\} .
\end{aligned}
$$

It is evident from (B.6) and (B.7) that $\mu_{X Y^{*}}=\left[\mu_{X^{*} Y}\right]^{*}$. We use the assumption that one spatial frequency is independent from the other, that is, correlation term at two different spatial frequencies is zero. Using (B.7), (B.6), and (B.5) into (B.3) we have

$$
\begin{aligned}
\mu_{X}^{k+1}(\bar{f}, \bar{f})= & \alpha^{2} \mu_{X}^{k}(\bar{f}, \bar{f}) \\
& +\beta^{2} \sum_{v}|H(v)|^{2}\left|U^{k}(v)\right|^{2} \mu_{X}^{k}(\bar{f}-v, \bar{f}-v) \\
& +2 \alpha \beta \sum_{v} \operatorname{Re}\left(H^{*}(v) U^{k}(v)\right) E\left(\left|X^{k}(\bar{f})\right|^{2}\right) \\
& -2 \alpha \beta \sum_{v} \operatorname{Re}\left(H^{*}(v) U^{k}(v)\right)\left|E\left(X^{k}(\bar{f})\right)\right|^{2},
\end{aligned}
$$

where Re denotes the real part of complex quantity.

\section{ABBREVIATIONS}

MAPE: maximum a Posteriori with entropy prior;

AAMAPE: adaptively accelerated MAPE;

RMSE: root mean square error;

SNR: $\quad$ signal-to-noise ratio;

PSF: point spread function.

\section{ACKNOWLEDGMENTS}

This work was supported by the Dual Use Center through the contract at Gwangju Institute of Science and Technology and by the BK21 program in the Republic of Korea.

\section{REFERENCES}

[1] P. A. Jansson, Deconvolution of Images and Spectra, Academic Press, New York, NY, USA, 1997.

[2] A. K. Katsaggelos, Digital Image Restoration, Springer, New York, NY, USA, 1989.

[3] A. K. Jain, Fundamentals of Digital Image Processing, PrenticeHall, Englewood Cliffs, NJ, USA, 2003. 
[4] A. S. Carasso, "Linear and nonlinear image debluring: a documented study," SIAM Journal on Numerical Analysis, vol. 36, no. 6, pp. 1659-1689, 1999.

[5] A. K. Katsaggelos, J. Biemond, R. W. Schafer, and R. M. Mersereau, "A regularized iterative image restoration algorithm," IEEE Transactions on Signal Processing, vol. 39, no. 4, pp. 914-929, 1991.

[6] F.-C. Jeng and J. W. Woods, "Compound Gauss-Markov random fields for image estimation," IEEE Transactions on Signal Processing, vol. 39, no. 3, pp. 683-697, 1991.

[7] R. Molina, A. K. Katsaggelos, J. Mateos, A. Hermoso, and C. A. Segall, "Restoration of severely blurred high range images using stochastic and deterministic relaxation algorithms in compound Gauss-Markov random fields," Pattern Recognition, vol. 33, no. 4, pp. 555-571, 2000.

[8] J. Zerubia, A. Jalobeanu, and Z. Kato, "Markov random fields in image processing application to remote sensing and astrophysics," Journal de Physique IV, vol. 12, no. 1, pp. 117$136,2002$.

[9] M. Nikolova, "Thresholding implied by truncated quadratic regularization," IEEE Transactions on Signal Processing, vol. 48, no. 12, pp. 3437-3450, 2000.

[10] E. S. Meinel, "Maximum-entropy image restoration: lagrange and recursive techniques," Journal of the Optical Society of America A, vol. 5, no. 1, pp. 25-29, 1988.

[11] R. Narayan and R. Nityananda, "Maximum entropy image restoration in astronomy," Annual Review of Astronomy and Astrophysics, vol. 24, pp. 127-170, 1986.

[12] J. L. Starck, E. Pantin, and F. Murtagh, "Deconvolution in astronomy: a review," Publications of the Astronomical Society of the Pacific, vol. 114, no. 800, pp. 1051-1069, 2002.

[13] J. M. Bioucas-Dias, "Bayesian wavelet-based image deconvolution: a GEM algorithm exploiting a class of heavy-tailed priors," IEEE Transactions on Image Processing, vol. 15, no. 4, pp. 937-951, 2006.

[14] R. C. Puetter, T. R. Gosnell, and A. Yahil, "Digital image reconstruction: deblurring and denoising," Annual Review of Astronomy and Astrophysics, vol. 43, pp. 139-194, 2005.

[15] M. Nikolova, "Local strong homogeneity of a regularized estimator," SIAM Journal on Applied Mathematics, vol. 61, no. 2, pp. 633-658, 2000.

[16] D. S. C. Biggs and M. Andrews, "Acceleration of iterative image restoration algorithms," Applied Optics, vol. 36, no. 8, pp. 1766-1775, 1997.

[17] L. Kaufman, "Implementing and accelerating the EM algorithm for positron emission tomography," IEEE Transactions on Medical Imaging, vol. 6, no. 1, pp. 37-51, 1987.

[18] H. M. Adorf, R. N. Hook, L. B. Lucy, and F. D. Murtagh, "Accelerating the Richardson-Lucy restoration algorithm," in Proceedings of the 4th ESO/ST-ECF Data Analysis Workshop, pp. 99-103, Garching, Germany, May 1992.

[19] T. J. Holmes and Y.-H. Liu, "Acceleration of maximumlikelihood image restoration for fluorescence microscopy and other noncoherent imagery," Journal of the Optical Society of America A, vol. 8, no. 6, pp. 893-907, 1991.

[20] D. S. C. Biggs and M. Andrews, "Conjugate gradient acceleration of maximum-likelihood image restoration," Electronics Letters, vol. 31, no. 23, pp. 1985-1986, 1995.

[21] R. G. Lane, "Methods for maximum-likelihood deconvolution," Journal of the Optical Society of America A, vol. 13, no. 6, pp. 1992-1998, 1996.

[22] W. H. Press, S. A. Teukolsky, W. T. Vetterling, and B. P. Flannery, Numerical Recipes in C, Cambridge University Press, Cambridge, UK, 2nd edition, 1992.
[23] J.-A. Conchello, "Superresolution and convergence properties of the expectation-maximization algorithm for maximumlikelihood deconvolution of incoherent images," Journal of the Optical Society of America A, vol. 15, no. 10, pp. 2609-2619, 1998.

[24] E. T. Jaynes, "On the rationale of maximum-entropy methods," Proceedings of the IEEE, vol. 70, no. 9, pp. 939-952, 1982.

[25] B. R. Frieden, "Restoring with maximum likelihood and maximum entropy," Journal of the Optical Society of America, vol. 62, no. 4, pp. 511-518, 1972.

[26] J. Skilling and S. F. Gull, "The entropy of an image," Proceedings of the American Mathematical Society, vol. 14, pp. 167-189, 1984.

[27] J.-L. Starck, F. Murtagh, P. Querre, and F. Bonnarel, “Entropy and astronomical data analysis: perspectives from multiresolution analysis," Astronomy \& Astrophysics, vol. 368, no. 2, pp. 730-746, 2001.

[28] H. J. Trussell, "The relationship between image reconstruction by the maximum a posteriori method and maximum entropy method," IEEE Transactions on Acoustics, Speech, and Signal Processing, vol. 28, no. 1, pp. 114-117, 1980.

[29] N. Vincent, A. Seropian, and G. Stamon, "Synthesis for handwriting analysis," Pattern Recognition Letters, vol. 26, no. 3, pp. 267-275, 2005.

[30] K. Goebel and W. A. Kirk, Topics in Metric Fixed Point Theory, Cambridge University Press, Cambridge, UK, 1990.

[31] J. G. Proakis and D. G. Manolakis, Digital Signal Processing, Pearson Education, Delhi, India, 3rd edition, 2004.

[32] J. M. Bioucas-Dias, "Bayesian wavelet-based image donvolution: a GEM algorithm exploiting a class of heavy-tailed priors," IEEE Transactions on Image Processing, vol. 15, no. 4, pp. 937-951, 2006.

[33] R. Neelamani, H. Choi, and R. Baraniuk, "ForWaRD: fourierwavelet regularized deconvolution for ill-conditioned systems," IEEE Transactions on Signal Processing, vol. 52, no. 2, pp. 418-433, 2004.

[34] V. Katkovnik, K. Egiazarian, and J. Astola, "A spatially adaptive nonparametric regression image deblurring," IEEE Transactions on Image Processing, vol. 14, no. 10, pp. 14691478, 2005.

[35] E. Veklerov and J. Llacer, "Stopping rule for the MLE algorithm based on statistical hypothesis testing," IEEE Transactions on Medical Imaging, vol. 6, no. 4, pp. 313-319, 1987.

[36] S. Ghael, A. M. Sayeed, and R. G. Baraniuk, "Improved wavelet denoising via empirical Wiener filtering," in Wavelet Applications in Signal and Image Processing V, vol. 3169 of Proceedings of SPIE, pp. 389-399, San Diego, Calif, USA, July 1997. 